\title{
Does age play a role in fever and neutropenia events and complications: A comparison of adolescents versus younger children with cancer at a tertiary care pediatric hospital, a pilot project
}

\author{
Nardin Kirolos ${ }^{1}$, Kenneth Tang ${ }^{2}$, and Lesleigh Abbott ${ }^{1}$ \\ ${ }^{1}$ Children's Hospital of Eastern Ontario \\ ${ }^{2}$ Children's Hospital of Eastern Ontario Research Institute
}

January 28, 2022

\begin{abstract}
Background: Adolescents and young adults with cancer (AYA) are a complex group of patients. The development of fever and neutropenia $(\mathrm{FN})$ is a potentially lethal complication of chemotherapy. Risk stratification of patients with FN has become increasingly valuable allowing for early intervention and for the use of lower intensity treatments. There are risk stratification guidelines that exist, but most are validated in young children with cancer (YCWC). AYA are frequently shown to have more numerous and severe side effects from chemotherapy. This study aimed to identify whether age contributes to the incidence and severity of FN. Procedure: Patients diagnosed with a malignancy in a 5-year period at our institution were included from ages 0-18 yrs. We reviewed details of their FN events, including duration of hospital admission, source (bacterial/fungal), PICU admission and duration, positive blood cultures and mortality. Results: Adolescents with cancer (AWC) had a trend of being 1.56 times more likely to have FN events (CI 95\% 0.936-2.622, $\mathrm{p}=0.087$ ). Assessment of the duration of PICU stay showed that AWC were 4.9 times more likely to have longer admissions (CI 95\% 0.998-24.067, p=0.050). There was no significant difference between the two groups in the rate of PICU admission, positive cultures, identification of a bacterial or fungal source, hospital admission duration or mortality. Conclusions: This study demonstrated a trend towards AWC being more likely to develop FN events. When such events occur in this group, the severity of them may be heightened as evidenced by longer duration of PICU admission.

\section{Introduction}

Adolescents and young adults with cancer (AYA) are commonly defined as those who fall within the age range of 15-29 years, with certain institutions extending this definition to include those up to 39 years of age. ${ }^{1,2}$ This groups faces numerous healthcare challenges that disproportionately impact their overall health outcomes and well-being compared to their pediatric and adult counterparts with the same diagnoses. ${ }^{1,2}$ Some of these disparities in care arise from their unique biologic, epidemiologic, and psychosocial factors in association with their underrepresentation in clinical trials. ${ }^{3-5}$ AYA have additionally been noted to suffer more side effects to therapy compared to younger children. ${ }^{5,6}$ Additional consideration must also be given to the discontinuity of care faced by AYA who are often treated at pediatric institutions until 18 years of age then transitioned to adult institutions beyond this, creating both physical and academic barriers to studying this population. Given the varying treatment protocols and approach to the management of complications between adult and pediatric institutions, the treatment of AYA is overall less uniform. All these aspects have contributed to the lag in improvement in cancer mortality for the AYA population, in contrast to the more drastic improvements made in younger children with cancer (YCWC). ${ }^{5,6}$ These unique factors highlight the need for further recognition of cancer associated risks in order to limit adverse health outcomes and improve overall survival for this group, by creating more standardized treatment guidelines. ${ }^{3-5}$
\end{abstract}


A common oncologic emergency is fever and neutropenia (FN) which is associated with increased morbidity and mortality ${ }^{7-9}$ The presence of neutropenia can result in an increased risk of life-threatening infections and chemotherapy dose reductions and delays, which may negatively impact treatment outcomes. ${ }^{10,11}$ Given that about half of the pediatric oncologic population develop at least one episode of $\mathrm{FN}$ when treated with chemotherapy, routine emergency hospitalization upon presentation and empirical antibiotic treatment has significantly reduced mortality over the years to around $1 \% .{ }^{12}$ However, FN presentations vary among patients with delayed recognition resulting in worse outcomes prompting the need for improvements in patient risk assessments. ${ }^{13,14}$ The effects of neutropenic sepsis have also been shown to more severely impact the AYA population, who are twice as likely to die from this complication in comparison with other age groups. ${ }^{15}$

Risk stratification strategies and clinical decision rules for presentations of FN have been adopted internationally in order to provide appropriate individualized treatment for patients. ${ }^{16}$ Various risk factors have been identified in predisposing patients to the development of FN, some of which include chemotherapy type and dose intensity, advanced age, comorbidities, elevated C-reactive protein, hypotension, leukemia as the cancer type, thrombocytopenia, and acute malnutrition. ${ }^{17,18}$ For patients who meet criteria of low risk, a reduced intensity treatment regimen as an outpatient, has been shown to be safe, improve quality of life and reduce costs of care. ${ }^{19}$ However, such stratification guidelines are based on young pediatric patients, rather than AYA and even within such regulations there remains an ongoing uncertainty as to the safety and efficacy of their applicability in all clinical settings. ${ }^{19,20}$

Due to the uncertainty of these risk indices, treatment of FN with intravenous antibiotics, irrespective of underlying risk, is most common. ${ }^{21,22}$ Further investigations are required to identify the feasibility of using risk factors, such as patient age, to risk stratify patients presenting with FN in order to further advance management, risk assessment and even consideration of preventative measures for cancer patients with this risk factor.

The main objective of this study is to assess the difference in quantity of FN presentations and severity between adolescents with cancer (AWC) and younger children with cancer (YCWC) who present to our pediatric institution. Given that our institution only treats patients up to 18 years of age, our study group of focus will be the AWC. The results of this study will be particularly pertinent to pediatric oncology clinicians who typically treat up to 18 years of age in their institutions.

The primary aim of this retrospective chart review was to compare the proportion of AWC to the proportion of YCWC who experienced FN following initial malignancy diagnosis to determine whether age is a risk factor for FN events. The secondary objective of this study was to identify the differences in severity of FN events in AWC and YCWC based on secondary variables including the duration of hospitalization, incidence of a bacterial or fungal causes of the event, incidence of a resulting PICU admissions, duration of PICU admission, incidence of positive blood cultures and incidence of mortality due to FN.

\section{Methods}

We conducted a retrospective chart review of all patients diagnosed with a malignancy from January 1 , 2013, to January 1, 2018 at the Children's Hospital of Eastern Ontario (CHEO). CHEO is a tertiary care, medium size institution in Ottawa, Canada that treats children from age 0-18 years old. This study was approved by the Research Ethics Board at CHEO. All malignancy types were included in the study, with no age restriction. Patients who presented with a relapse from a previous cancer diagnosis or who did not receive chemotherapy as part of their treatment regimen were excluded as their risk factors for severity and number of episodes of FN were felt to be greater or lesser respectively.

For each enrolled patient, data on their sex, date of diagnosis, age at the time of diagnosis, primary oncologic diagnosis, treatment protocol(s), date of treatment completion, and current mortality status were collected from their health record. A FN event was identified at any time when the patient presented with an absolute neutrophil count (ANC) of less than 500 cells $/ \mathrm{mm}^{3}$ and fever according to institutional definition. Fever was defined as an axillary temperature of $38^{\circ} \mathrm{C}$ or greater measured once or a temperature between 37.6- 
$37.9^{\circ} \mathrm{C}$ measured twice at timepoints an hour apart. If measured orally, patients were considered febrile at $38.5^{\circ} \mathrm{C}$ or greater measured once, or a temperature between $38.0-38.4^{\circ} \mathrm{C}$ measured twice after an hour's time. For each FN episode, information was collected on the date of presentation and age of the patient at that time. Severity was assessed by consideration of our secondary outcomes which are the duration of hospital admission, the discovery of a source for the FN (bacterial/fungal), a resulting PICU admission and duration, the presence of a positive blood culture and mortality from the FN event. Multiple FN episodes per patient were counted and assessed. The hospital and PICU length of stay variables were only calculated based on admissions where patients presented to the emergency department with FN, rather than recorded FN events that developed during a hospital stay for an alternate admitting diagnosis.

Patient characteristics and outcomes were described using descriptive statistics. Continuous variables were summarized as means, standard deviations, and range, and categorical variables were summarized as frequencies and percentages. Due to the varying treatment durations and therefore the varied risk for the number of FN events during the course of individual patient treatments, the rate of FN was also quantified and described, which was calculated as the total frequency of each type of FN over the treatment duration in years. Patients were sub-categorized by age into the AWC if they were 15 years or older up to 18 years old at the time of diagnosis or in the YCWC group if they had an age of less than 15 at the time of diagnosis. Quasi-poisson models were fitted to assess the relationship between age groups and FN complications while controlling for key covariates of gender and diagnosis type (7 levels, leukemia set as reference category). In these models, the number of FN events was specified as the outcome while the natural logarithm of treatment duration was specified as an offset parameter. Effect sizes were expressed in terms of adjusted rate ratios and associated $95 \%$ confidence intervals $(95 \% \mathrm{CI})$. All analyses were conducted with $R$ version 4.0.5. ${ }^{23}$

Results

Between January 2013 and January 2018, 379 children and adolescents were diagnosed with a malignancy; 265 of which were included in the analysis (Fig. 1). Data was collected for each enrolled patient until June 2019. There were $14.3 \%$ of patients with a minimum age of 15 years old who were classified under the AWC group. The median age at diagnosis was 6.80 (IQR, 3.2-12.86 years) for all patients, 16.46 (IQR, 15.5-17.38) for the AWC and 5.93 (IQR, 2.68-9.88) for the YCWC. Over half the patients were male (55.8\%). Patients had varying cancer diagnoses of which the majority were leukemias (41.1\%), followed by lymphomas (18.5\%), primary brain tumours $(15.1 \%)$, neuroblastoma $(6.4 \%)$, sarcomas $(6.0 \%)$, Wilms tumour $(4.9 \%)$ and germ cell tumours (1.9\%). The remaining diagnoses $(6.0 \%)$ were grouped together due to their rarer occurrence. The majority of patients $(81.9 \%)$ were treated with one chemotherapy protocol. The treatment duration (mean $\pm \mathrm{SD}$ ) for the AWC was 1.01 \pm 0.93 years and $1.85 \pm 1.39$ for the YCWC Additional demographic and treatment characteristics are summarized in Table 1.

In the AWC group, the average FN event occurrence was 1.71 (SD 2.42), with 10.5\%, $13.2 \%$ and $15.8 \%$ of patients having one, two or three FN episodes respectively. In this group, $47.4 \%$ of patients did not have any FN events. The maximum number of 10 episodes was had by one patient $(2.6 \%)$. In contrast, the YCWC patients had an average of 1.84 (SD 1.91) FN events. In this group, 36.6\% of patients did not have any FN events, while the majority of those who had an event had only one episode (16.7\%). The remaining patients had two $(11.5 \%)$ or three $(13.7 \%)$ episodes. One patient $(0.4 \%)$ had the maximal number of nine episodes in this age group. The rate of $\mathrm{FN}$ among $\mathrm{AWC}$ and $\mathrm{YCWC}$ was $1.86 \pm 2.61$ and $1.58 \pm 2.69$ respectively (Fig. 2) yielding a rate ratio of 1.567 [(CI 95\% 0.936, 2.622), $p=0.087$ ] for FN events of AWC compared to YCWC, adjusted for covariates of gender and all diagnosis types.

In evaluation of the secondary outcomes for severity of FN episodes, the AWC had an average of 0.16 (SD 0.37 ) bacterial episodes with only $15.8 \%$ of the group having one such documented episode. They averaged 0.05 (SD 0.23) fungal episodes, experienced by only $5.3 \%$ of the group. The YCWC group had a mean average of 0.27 (SD 0.67) bacterial episodes, with the majority of those confirmed to have a bacterial infection having only one episode $(12.8 \%)$, but a minority having two $(2.2 \%)$, three $(2.6 \%)$, or four $(0.4 \%)$ episodes. In the YCWC group, no episodes were related to a fungal infection. In comparing rates of the AWC to YCWC, for the bacterial episodes they were 0.19 (SD 0.61) to 0.20 (SD 0.71) and for the fungal causes they were 0.02 
(SD 0.09) to 0.0 (SD 0).

When combining the identification of bacterial and fungal sources during all FN episodes, those in the AWC group had an overall average number of 0.21 (SD 0.47), with $18.4 \%$ of the patients having at least one of these events. The YCWC had a mean of 0.27 (SD 0.67), with $18.1 \%$ of the group having between one and four occurrences of this severity index. The rate for the AWC group was 0.21 (SD 0.61) compared to the YCWC group which was 0.20 (0.71). When collating all the findings to assess for the role that age has on the severity of $\mathrm{FN}$ presentations denoted by the identification of bacterial and/or fungal sources, a rate ratio of 1.258 [(CI 95\% 0.423, 3.736), $p=0.680$ ] for the AWC compared to the YCWC was found (Fig. 3A).

In examination of severity based on the incidence of positive blood cultures, either bacteremia or fungemia, during FN presentations, the AWC group averaged 0.21 (SD 0.47) with $18.4 \%$ of the group having 1 or 2 positive blood cultures. The rate of this index was 0.21 (SD 0.61). For the YCWC, they had an average of 0.30 (SD 0.68) positive blood cultures. In the YCWC group, $20.7 \%$ of the patients had a minimum of one positive blood culture and the rate for the group was found to be 0.22 (SD 0.69). As such, the rate ratio for the finding of a positive blood culture in AWC vs. YCWC was 1.155 [(CI 95\% 0.421, 3.172), $p=0.780$ ] (Fig. $3 \mathrm{~A})$.

Further severity assessment based on time spent in hospital for patients with the main admitting diagnosis of FN revealed that the AWC spend an average of 10.11 (SD 16.41) days in hospital, while the YCWC spent 14.84 (SD 23.54) days. The AWC had a rate of 10.70 (SD 18.43), while the YCWC had a rate of 11.64 (SD 27.53) (Fig. 3B). Comparison of the time spent by both groups yielded a ratio of 1.136 [(CI 95\% 0.536, $2.405, p=0.740]$.

Evaluation of the severity index of PICU admission revealed that for the AWC, $5.3 \%$ of patients in this age group had one PICU admission. In contrast, $3.5 \%$ of patients under 15 years of age had at least one PICU admission. Analysis of the duration of PICU admission revealed that the average duration of stay for the AWC in PICU was 0.16 (SD 0.82) days with a rate of 0.31 (SD 1.86) and 0.12 (SD 0.97) days for the YCWC with a rate of 0.13 (SD 1.21) (Fig. 3B). The rate ratio of the AWC to the YCWC was statistically significant at $4.900[(\mathrm{CI} 95 \% 0.998,24.067), p=0.050]$.

Amongst all patients enrolled in the study, there was $10.9 \%$ of mortality, made up entirely of the YCWC group, but no death was attributable solely to a FN event.

\section{Discussion}

We observed that compared to YCWC, AWC show a trend towards being 1.6 times more likely to develop FN. Additionally, when such events occur in this age group, the severity of them may be heightened as shown by the statistically significant finding that they are 4.9 times more likely to have a longer admission to PICU, when admission is secondary to their FN presentation.

Given our findings that suggest that age may play a role in FN, and known discoveries that AYA have been found to be twice as likely to die of neutropenic sepsis compared to other age groups, it is imperative that this group of patients is prioritized for their own set of guidelines that account for age in risk stratification. ${ }^{15}$ Risk assessment guidelines are essential in establishing a standard for the extent of necessary investigations, type of empirical antibiotic therapy to be used with regards to the formulation in which they are provided, duration of antibiotic therapy, neutrophil recovery necessary prior to discharge or discontinuation of antibiotics, the need for prophylaxis throughout chemotherapy treatment and determination of whether treatment is conducted inpatient or outpatient. ${ }^{24}$

There are numerous risk stratification tools (i.e. Rackoff ${ }^{25}$, Alexander $^{26}, \mathrm{PINDA}^{27}, \mathrm{SPOG}^{28}, \mathrm{Ammann}^{29}$ and $\mathrm{MASCC}^{30}$ ) that have been developed to better stratify patients as low risk based on the likelihood of severe outcomes as a result of FN. Although there have been numerous studies, such as Haeusler et al. (2020), which have validated many of these existing risk stratification tools, the median age of the study patients on which these tools are validated typically falls within the YCWC population with a median age of 5-6 years of age. ${ }^{19}$ Additionally, countless studies have shown that all rules perform inadequately in their 
ability to appropriately discriminate AYA patients as low or high risk. ${ }^{20}$ The inconsistent performance of these tools across cohorts has left various panels aimed at developing guidance statements to advise that individual sites select which rules to use based on their available resources and population data, rather than evidence based superiority of rules. ${ }^{31,32}$ The findings of this study do not suggest that all previous risk factors studied in the YCWC group be abandoned for the AYA population; they do however support the implementation of age into such existing guidelines in order to individualize them to this unique population.

Our findings which suggest that adolescence as an individual variable may increase both the incidence and severity of FN in the pediatric oncological population provides an opportunity in which age can be used to risk stratify younger patients with cancer to a lower risk group. ${ }^{20}$ Similar approaches to this have been shown to be effective in the adult oncology population who present with FN but are classified as low risk. ${ }^{33}$ These results also bring up the consideration of higher thresholds for prophylaxis antimicrobials for AWC patients being a consideration in future research as well as clinically. Further refinement of risk factors could help decrease the incidence of these mentioned events. With the addition of our findings that PICU admission duration in the AWC is significantly longer, early identification and treatment of this age group in particular may not only contribute to decreasing the likelihood of serious complications, but will also be more cost effective for institutions.

This study is limited by its retrospective design and the associated restrictions. Additionally, given that CHEO only treats patients to an upper limit age of 18 years old, our study focused on the AWC population, rather than the broader AYA group. In future projects, we plan on working with our adult oncology teams to expand the AWC group to include patients up to 29 years of age, in order to have our findings more applicable to the entire AYA group. However, our findings are pertinent to the majority of pediatric oncology clinicians who typically treat up to the age of 18 years old at their institutions. Our findings are preliminarily assessing patients with cancer irrespective of their diagnosis or treatment regimen. Future studies may further assess the role of how the known disproportionate incidence of FN with various malignancies and different chemotherapy regimens also contribute either similarly or differently to patients with cancer of varying ages.

In conclusion, this study highlights that differences in FN event occurrence and severity exist between AWC and YCWC. Further refinement of risk factors is necessary in order to better risk stratify oncology patients and develop more appropriate, individualized treatment algorithms.

\section{Conflicts of Interest}

All authors declare no conflicts of interest.

\section{References}

1. Ramphal R, Aubin S, Czaykowski P, et al. Adolescent and young adult cancer: principles of care. Curr Oncol 2016;23(3):204-209.

2. Aben K, van Gaal C, van Gils NA, et al. Cancer in adolescents and young adults (15-29 years): a population-based study in the Netherlands 1989-2009. Acta Oncol 2012;51(7):922-33.

3. Fernandez C, Fraser GAM, Freeman C, et al. Principles and recommendations for the provision of healthcare in Canada to adolescent and young adult-aged cancer patients and survivors. J Adoles and Young Adult Onc 2011;1(1):53-59.

4. Bleyer A. Older adolescents with cancer in North America deficits in outcome and research. Pediatr Clin North Am 2002;49:1027-42.

5. Bleyer A, O'Leary M, Barr R, et al. Cancer Epidemiology in Older Adolescents and Young Adults 15 to 29 Years of Age, Including SEER Incidence and Survival: 1975-2000. National Cancer Institute: Bethesda, NIH Pub 2006;No. 06-5767.

6. Freyer DR, Felgenhauer J, Perentesis J; COG Adolescent and Young Adult Oncology Discipline Committee. Children's Oncology Group's 2013 blueprint for research: adolescent and young adult oncology. Pediatr Blood Cancer 2013;60(6):1055-8.

7. Meckler G, Lindemulder S. Fever and neutropenia in pediatric patients with cancer. Emerg Med Clin North Am 2009;27(3):525-44. 
8. Lustberg MB. Management of neutropenia in cancer patients. Clin Adv Hematol Oncol 2014;10(12):825-26.

9. Badr M, Hassan T, Sakr H, et al. Chemotherapy-induced neutropenia among pediatric cancer patients in Egypt: risks and consequences. Mol Clin Oncol 2016;5(3):300-206.

10. Ammann R, Tissing W, Phillips B. Rationalizing the approach to children with fever in neutropenia. Curr Opin Infect Dis 2012;25:258-265.

11. Burutaran M, Guadagna1 R, Grille S, et al. Results of high-risk neutropenia therapy of hematologyoncology patients in a university hospital in Uruguay. Rev Bras Hematol Hemoter 2015;37:28-33.

12. Zermatten M, Koenig C, von Allmen A, et al. Episodes of fever in neutropenia in pediatric patients with cancer in Bern, Switzerland, 1993-2012. Sci Data 2019;6:180304.

13. Weycker D, Barron R, Kartashov A, et al. Incidence, treatment and consequences of chemotherapyinduced febrile neutropenia in the inpatient and outpatient settings. J Oncol Pharm Pract 2014;20:190198.

14. Freifeld A, Bow E, Sepkowitz K, et al. Clinical practice guideline for the use of antimicrobial agents in neutropenic patients with cancer: 2010 update by the Infectious Diseases Society of America. Clin Infect Dis 2011;52:e56-e93.

15. National Institute for Health and Clinical Excellence. Neutropenic sepsis: prevention and management of neutropenic sepsis in cancer patients. Clinical guideline 151.2012.

16. Delebarre M, Tiphaine A, Martinot A, et al. Risk-stratification management of febrile neutropenia in pediatric hematology-oncology patients: Results of a French nationwide survey. Pediatr Blood Cancer 2016;63(12):2167-72.

17. Family L, Li Y, Chen L, et al. Risk factors for febrile neutropenia in cancer patients treated with chemotherapy. J Clin Oncol 2016;34(15):suppl6559.

18. Asturias E, Corral J, Quezada J. Evaluation of six risk factors for the development of bacteremia in children with cancer and febrile neutropenia. Curr Oncol 2010;17(2):59-63.

19. Haeusler G, Thursky K, Slavin M, et al. Risk stratification in children with cancer and febrile neutropenia: a national, prospective, multicentre validation of nine clinical decision rules. The Lancet 2020;18:100220.

20. Phillips S, Bhuller K, Sung L, et al. Risk stratification in febrile neutropenic episodes in adolescent/young adult patients with cancer. Eur. J. Cancer 2016;64:101-06

21. Herd F, Bate J, Chisholm J, et al. Variation in practice remains in the UK management of pediatric febrile neutropenia Arch Dis Child. 2016;101:410-411.

22. Phillips R, Lehrnbecher T, Alexander S, et al. Updated systematic review and meta-analysis of the performance of risk prediction rules in children and young people with febrile neutropenia. PLoS One 2012;7:e38300.

23. R: A language and environment for statistical computing [R Foundation for Statistical Computing]. Vienna, Austria: R Core Team; 2021.

24. Freifeld G, Bow E, Sepkowitz K, et al. Clinical Practice Guideline for the Use of Antimicrobial Agents in Neutropenic Patients with Cancer: 2010 Update by the Infectious Diseases Society of America. Clin Infect Dis 2011;52(4):e56-e93.

25. Rackoff W, Gonin, R, Robinson, C, Kreissman, S, Breitfeld P. Predicting the risk of bacteremia in children with fever and neutropenia. J Clin Oncol 1996;14(3): 919-24.

26. Alexander S, Wade K, Hibberd P, Parsons S. Evaluation of risk prediction criteria for episodes of febrile neutropenia in children with cancer. J Pediatr Hematol Oncol 2002;24:38-42.

27. Santolaya M, Alvarez A, Becker A, et al. Prospective, multicenter evaluation of risk factors associated with invasive bacterial infection in children with cancer, neutropenia, and fever. J Clin Oncol 2001;19:3415-3421.

28. Agyeman P, Aebi C, Hirt A, et al. Predicting bacteremia in children with cancer and fever in chemotherapy-induced neutropenia: results of the prospective multicenter SPOG 2003 FN study. Pediatr Infect Dis J 2011;30:e114-119.

29. Ammann R, Bodmer N, Hirt A, et al. Predicting adverse events in children with fever and 
chemotherapy-induced neutropenia: the prospective multicenter SPOG 2003 FN study. J Clin Oncol 2010;28(12):2008-14.

30. Klastersky J, Paesmans M, Rubenstein E, et al. The Multinational Association for Supportive Care in Cancer risk index: A multinational scoring system for identifying low-risk febrile neutropenic cancer patients. J Clin Oncol 2000;18(16):3038-51.

31. Melgar M, Reljic T, Barahona G, et al. Guidance statement for the management of febrile neutropenia in pediatric patients receiving cancer-directed therapy in Central America and the Caribbean. JCO Glob Oncol 2020;6:508-17.

32. Lehrnbecher T, Robinson P, Fisher B, et al. Guideline for the management of fever and neutropenia in children with cancer and hematopoietic stem-cell transplantation recipients: 2017 update. J Clin Oncol 2017;35(18):2082-94.

33. Teh BW, Brown C, Joyce T, Worth LJ, Slavin MA, Thursky KA. Safety and cost benefit of an ambulatory program for patients with low-risk neutropenic fever at an Australian centre. Support Care Cancer 2018;26(3):997-100310.

Please note: The data that support the findings of this study are available on request from the corresponding author. The data are not publicly available due to privacy or ethical restrictions.

Legends:

FIGURE 1 Flow of patients in the study.

FIGURE 2 Rate of FN events in the AWC and YCWC where rate is calculated as the mean frequency of FN events over the mean treatment duration of the malignancy in years. SD error bars plotted.

FIGURE 3. Rate of secondary outcomes of severity of FN episodes in the AWC and YCWC. SD error bars plotted. Rate is calculated as (A) the mean frequency of identified bacterial and fungal infections in a FN episode and frequency of positive blood cultures (either bacteremia or fungemia) over the mean treatment duration of the malignancy in years. In (B), rate is calculated as the mean duration of the hospital admission in days in those admitted for FN over the mean treatment duration of the malignancy in years.

Hosted file

TABLES - FN .docx available at https://authorea.com/users/457793/articles/554495-doesage-play-a-role-in-fever-and-neutropenia-events-and-complications-a-comparison-of-

adolescents-versus-younger-children-with-cancer-at-a-tertiary-care-pediatric-hospital-apilot-project 